\title{
Plasmonic band gap engineering of plasmon-exciton coupling
}

\author{
Ertugrul Karademir, ${ }^{1}$ Sinan Balci, ${ }^{2}$ Coskun Kocabas, ${ }^{1}$ and Atilla Aydinli ${ }^{1, *}$ \\ ${ }^{1}$ Department of Physics, Bilkent University, 06800 Ankara, Turkey \\ ${ }^{2}$ Department of Astronautical Engineering, University of Turkish Aeronautical Association, 06790 Ankara, Turkey \\ ${ }^{*}$ Corresponding author: aydinli@fen.bilkent.edu.tr
}

Received June 16, 2014; revised August 27, 2014; accepted August 29, 2014;

posted September 2, 2014 (Doc. ID 214154); published September 29, 2014

\begin{abstract}
Controlling plasmon-exciton coupling through band gap engineering of plasmonic crystals is demonstrated in the Kretschmann configuration. When the flat metal surface is textured with a sinusoidal grating only in one direction, using laser interference lithography, it exhibits a plasmonic band gap because of the Bragg scattering of surface plasmon polaritons on the plasmonic crystals. The contrast of the grating profile determines the observed width of the plasmonic band gap and hence allows engineering of the plasmonic band gap. In this work, resonant coupling between the molecular resonance of a J-aggregate dye and the plasmonic resonance of a textured metal film is extensively studied through plasmonic band gap engineering. Polarization dependent spectroscopic reflection measurements probe the spectral overlap occurring between the molecular resonance and the plasmonic resonance. The results indicate that plasmon-exciton interaction is attenuated in the band gap region along the grating direction. (c) 2014 Optical Society of America

OCIS codes: (050.2770) Gratings; (240.6680) Surface plasmons; (160.5293) Photonic bandgap materials.

http://dx.doi.org/10.1364/OL.39.005697
\end{abstract}

If a material is driven with an external high frequency electric field, the response of the atomic nuclei, owing to their larger mass, is considerably slower than that of electrons and hence a net polarization occurs. At frequencies lower than and in the vicinity of surface plasmon frequency, $\omega_{\mathrm{sp}}^{2}=\omega_{p}^{2} / 2$ (where $\omega_{p}$ is the plasma frequency), collective oscillation of surface electrons becomes relatively easier, forming surface plasmon modes. These modes are as energetic as photons with a considerable amount of momentum. Once the momentum mismatch is compensated, the incident photons can be coupled to these modes forming surface plasmon polaritons (SPPs). The resulting dispersion curve of the SPPs propagating along a planar metal dielectric interface is a continuous function of momentum and is given as [1]

$$
\omega_{\mathrm{SPP}}^{2}=\frac{k^{2}}{\mu_{0}}\left(\frac{1}{\varepsilon_{d}}+\frac{1}{\varepsilon_{m}}\right)
$$

Degeneracy of forward and backward propagating SPP modes at a given frequency can be lifted by patterning the planar surface with a periodic relief structure, such as a sinusoidal uniform grating pattern. [2] Under resonance, this pattern satisfies the conditions of plasmonic band gap formation and slows down [ $\underline{3}-\underline{5}]$ the SPPs at the two band edges. $[1,2,6]$ These band edges have been known for decades $[\overline{7}, \overline{8}]$ and have been studied theoretically and experimentally for their dependence on the grating surface profile [1-3, $\underline{3}-10]$, enhancement of Raman scattering [11], availability for signal feedback [12], role on photo luminescence [13], and interactions with J-aggregate complexes [14]. In addition, the group velocity of SPPs has been greatly decreased through plasmonic band gap engineering of coupled plasmonic cavities [15].

A model system for investigating light matter interactions is the coupled plasmon-exciton states, which are named plexcitons [16]. A mark of the strong coupling is the observation of Rabi splitting [16-22] because of the newly emerged in-phase and out-of-phase eigenmodes [16]. The energy difference between these modes is defined as

$$
\hbar \Omega_{R}=\sqrt{4 V^{2}-\left(\gamma_{p}-\gamma_{e}\right)^{2}}
$$

where $V \propto \vec{E} \cdot \vec{\mu}$, defines the energy transfer term between plasmons and excitons; $\gamma_{p}$ and $\gamma_{e}$ are damping terms related to plasmons and excitons [17]. The electric field in the energy transfer term is directly related to the amplitude or the number of plasmonic modes. At the band edges, because of the decreased group velocity and standing wave formation of SPP modes, the number of plasmonic modes also increases, causing a further enhancement compared to propagating modes. Recently, plexitonic states have been investigated on a variety of plasmonic structures such as flat metals, spherical nanoparticles, metal disks, metal nanowires, and metal prisms $[\underline{14}, \underline{16}-\underline{22}]$. However, plasmon-exciton coupling has not been studied using plasmonic band gap materials. Studies in this direction might significantly improve the performance of the plasmonic devices and enable understanding of the mechanism of the coherent energy transfer between metallic nanostructures and semiconductor nanostructures.

In this work, we study interaction between plasmons and excitons through band gap engineering of 1D metallic grating in $\Gamma$ direction, which has been achieved by varying the groove contrast of the metallic grating in the visible spectrum. This is a simple, yet effective, tool to engineer the band gap of the periodic plasmonic structures, altering the distribution of the plasmonic states. Using this passive tuning approach, we are able to adjust the distribution of plasmonic states and manipulate the coupling between SPPs and excitons. The plasmonic band gap engineering is schematically summarized in Fig. 1 . The depth of the grating groove determines the 
(a)

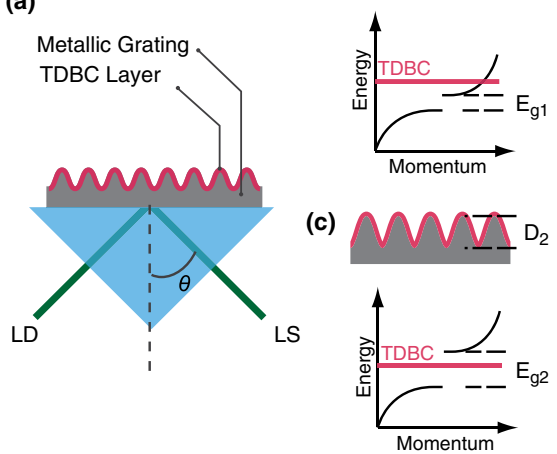

Fig. 1. (a) Schematic representation of the experimental setup. Samples are fabricated on glass slides and mounted on a prism with an index matching fluid, which is used to eliminate reflection losses associated with the glass-air interface and to ensure optical continuity between the prism and the glass slide. Thus the light from the light source (LS) effectively remains in the same medium inside the prism. (b), (c) Periodic corrugation along the metal dielectric interface introduces a band gap in the dispersion curve of the SPPs. The grating groove depth enables controlling of the $E_{\mathrm{pg}}$ manifested in the dispersion curve of the SPPs. The width of the band gap, $E_{\mathrm{pg}}$, depends on the grating groove depth and increases with it. The narrow absorption band of the aggregated TDBC dye is shown as a red line in the dispersion curve. Since the energy of the molecular resonance of the J-aggregate is constant, the $E_{\text {pg }}$ can be engineered to study plasmon-exciton interaction on different regions of the band gap. This kind of manipulation of energy levels requires the preparation of plasmonic crystals with varying groove depths.

width of the plasmonic band gap energy, $E_{\mathrm{pg}}$. Deeper grooves result in larger $E_{\mathrm{pg}}$. The molecular resonance of the dye remains at the same wavelength while, the $E_{\text {pg }}$ of the gratings is varied. Therefore, plasmon-exciton coupling can be studied at different parts (inside and outside) of the band gap. It should be added that spectral position of the band gap can also be altered by varying the periodicity of the grating structure; however the periodicity of the grating is fixed for all the gratings studied here. For directions other than the grating direction ( $\Gamma$ direction), 1D grating may not show a band gap. If the crystal is rotated $\pi / 2$ degrees with respect to the $\Gamma$ direction, then the dispersion behavior of the crystal becomes identical to that of the flat metal because the incident light does not experience a periodic corrugation; in between, the band gap will shift toward blue wavelengths. Hence, the results presented here are for $1 \mathrm{D}$ grating along $\Gamma$ direction and not for a full photonic band gap. [23]

Figure 1(a) shows the schematic representation of the experimental setup used for obtaining the reflectivity data recorded as a function of the photon wavelength and the incidence angle. Incident light is coupled to SPPs via prism coupling technique. Sinusoidal gratings have been fabricated using laser interference lithography. Glass substrates (3 mm thick) are first coated with an antireflection coating BARLi (AZ Chemicals) and then with a photosensitive polymer, S 1800-4 (MicroChem). Total thickness of the polymer layer is around $250 \mathrm{~nm}$. The sample is exposed in the lithography setup using the He-Cd laser operating at a wavelength of $325 \mathrm{~nm}$. Finally, a thin film of silver $(\sim 40 \mathrm{~nm})$ is thermally evaporated on the fabricated sinusoidal grating to support propagation of SPPs. The depth of the grating groove is adjusted by only varying the exposure time of the photopolymer. The surface profile and the groove depth of the gratings have been confirmed using an atomic force microscope. A cyanine dye, 5,5', 6,6'-tetrachloro-di-(4-sulfobutyl) benzimidazole-carbocyanine (TDBC) (Few Chemicals), forms J-aggregates in aqueous solutions at high concentrations. After dissolving the dye in water, it is mixed with a $1.5 \%$ Poly vinyl alcohol (PVA) solution in a 1:1 ratio to prepare $0.75 \%$ PVA. At high concentrations, individual dye molecules self-assemble in a head-to-tail fashion to form J-aggregates. J-aggregates have narrower absorption band shifted to the red region of the electromagnetic spectrum with respect to their monomer form. Ultrathin films of $1.25 \mathrm{mM}$ of J-aggregates ( $\sim 30 \mathrm{~nm})$ are spin-coated on the metal covered grating structures. SPPs are excited through a triangular BK7 glass prism in the Kretschmann configuration (KC). A tunable $p$-polarized monochromatic light source is used to probe the coupling. Reflection spectra are obtained by varying the wavelength of the incoming light and recording the reflected light intensity with a silicon photo-detector at each incidence angle. Finite-difference-time-domain (FDTD) simulations have been performed using commercial software package (Lumerical Solutions, Inc.). Sinusoidal grating structure of $\mathrm{SiO}_{2}$ glass substrate is covered with a thin $\mathrm{Ag}$ film. Bloch boundary conditions are applied along the lateral direction of the simulation window with one period width in order to simulate the infinite array of repeating patterns forming the plasmonic crystal. Optical constants of the used materials are taken from Palik [24]. J-aggregate dye is modeled as a Lorentz absorber whose resonance frequency is set at an absorption line of TDBC dye. The strength of the Lorentz absorber is obtained by fitting the optical parameter data acquired with an ellipsometer [17]. A light source is used for generating white light and a monitor is used for collection of the reflected light. The source and the monitor are positioned inside the glass substrate to mimic the KC. The spectrum obtained from the monitor is normalized with respect to the source. In this way, reflectivity spectra as a function of wavelength for each incidence angle that are similar to the experimental reflectivity spectra are constructed.

A sample of the resulting reflection map is shown in Fig. 2(a), which is obtained in a simulated KC. The separation between high and low frequency components are directly dependent on the groove depth of the grating and are marked as upper and lower band edges in Fig. 2(e). The $E_{\mathrm{pg}}$ for shallow grooves (up to $60 \mathrm{~nm}$ in depth) has been calculated in Refs. [6] and [1] with non-Hermitian and Hermitian eigenvalue problems set up with classical Maxwell equations. The calculations have shown that the $E_{\text {pg }}$ is approximately linearly proportional to the depth of the grooves.

In Fig. 2(f), normalized distribution of plasmonic states is shown for the bare gratings of varying groove depths with the same periodicity $(260 \mathrm{~nm})$. The distribution of the plasmonic states is calculated by 

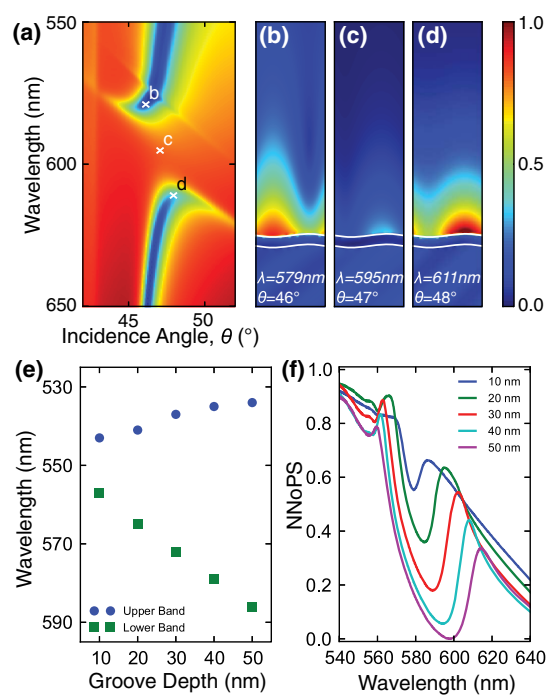

Fig. 2. (a) Band gap engineering has been performed by tuning the grating of $260 \mathrm{~nm}$ pitch groove depth. The reflection map showing the plasmonic band gap is obtained from a thin metal film coated on a sinusoidal grating, without the J-aggregate load, having a grating groove depth of $15 \mathrm{~nm}$. (b), (c), and (d) are the spatial profiles of electric field intensity averaged over time and recorded for critical regions on the reflection map. White lines mark the boundaries of the metal film. The lower part of the film is $\mathrm{SiO}_{2}$, and the upper part is a vacuum. (b) and (d) are the profiles for the upper (grating trough) and lower (grating peaks) band edges, respectively. (c) is the electric field profile inside the band gap region, which does not show any field; hence at this configuration, the interaction between surface plasmons and excitons is expected to be effectively minimized. (e) Wavelength position of the upper and lower band edges as a function of the grating groove depth simulated by using FDTD method is demonstrated. Band edges are determined by marking the reflection minima at each wavelength and then recording the lowest energy of the upper branch and highest energy of the lower branch. (f) NNoPS calculated for gratings with different groove depths.

$$
\operatorname{NNoPS}(\omega)=\int_{k_{\mathrm{MIN}}}^{k_{\mathrm{MAX}}} \mathrm{d} k \frac{[1-R(\omega, k)]}{R_{\mathrm{MAX}}},
$$

where $R(\omega, k)$ is the reflectivity of the incident light for a given frequency and momentum. Both in the experiment and in the simulations, convention is to adjust the momentum of light by varying the incidence angle. $k_{\mathrm{MAX}}$ and $k_{\mathrm{MIN}}$ are maximum and minimum spatial frequencies that can be obtained using the wavelength and incidence angles. The reflection map exemplified in Fig. 2(a) can be interpolated to the momentum space by using the relation $k_{\mathrm{SPP}}=k_{0} n_{\mathrm{PRISM}} \sin \theta$. After interpolation, normalized number of plasmonic states (NNoPS) for each wavelength or frequency is calculated with Eq. (3).

It should be noted that, there are still available plasmonic states inside the band gap. However, increasing the groove depth effectively deepens the minima in the NNoPS. This makes effective control of the plasmon-exciton coupling possible together with the coupling term introduced in Eq. (2). Flat band dispersion at the band edges that are because of the standing wave formation at the grating peaks and troughs can be observed as peaks in NNoPS. Finally, we note that apparent negative group index at the branches [Fig. 2(a)] is because of the small fraction of plasmons that are scattered back and propagating in the opposite direction. In Figs. 2(b) $-2(d)$, electric field profiles inside, and at the upper and the lower edges of the band gap are shown. Figures 2(b) and $2(\mathrm{~d})$ are the expected eigenmode profiles obtained from the standing wave solutions of such periodic corrugation. In addition, electric field intensity is greatly suppressed and nearly zero inside the band gap, Fig. 2(c).

Loading the samples of $280 \mathrm{~nm}$ grating pitch with the J-aggregate layer introduces an anti-crossing at the molecular resonance wavelength $(\sim 590 \mathrm{~nm})$. The band gap has been engineered by varying the grating groove depth from 5 to $15 \mathrm{~nm}$. The anti-crossing behavior at the upper branch of the band gap is clearly seen for the grating with the groove depth of $5 \pm 2 \mathrm{~nm}$ [Fig. 3(a)]. For the grating with the groove depth of $10 \pm 2 \mathrm{~nm}$, the lower branch of the anti-crossing is attenuated and diminished further when the grating groove depth is $15 \pm 2 \mathrm{~nm}$. This trend can also be observed in the FDTD simulations of the gratings as demonstrated in Fig. 3(b).
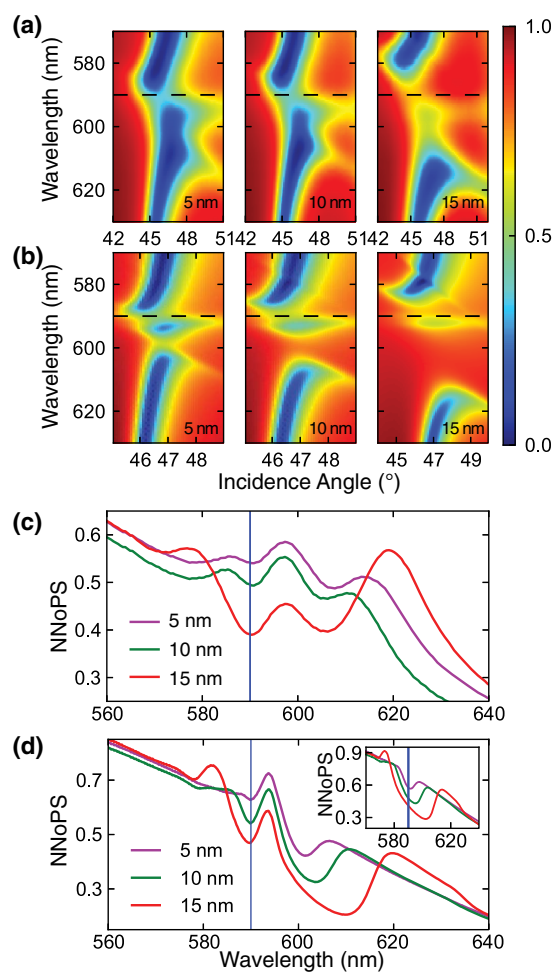

Fig. 3. (a) Experimentally obtained reflection maps for gratings with a $280 \mathrm{~nm}$ pitch. J-aggregate molecular resonance is marked with a dashed line at around $590 \mathrm{~nm}$. The $E_{\mathrm{pg}}$ is effectively enlarged by increasing the grating groove depth from 5 to $15 \mathrm{~nm}$, and thus the interaction between excitons and plasmons is attenuated or completely damped. For 5 and $10 \mathrm{~nm}$ groove depths, the anti-crossing behavior at $590 \mathrm{~nm}$ representing the strong coupling is clearly observed, where Rabi splitting energy is 40 and $50 \mathrm{meV}$, respectively. (b) FDTD simulation of the identical samples used in the experimental measurements confirms the observed results. (c) and (d) NNoPS obtained from (c) experimental and (d) simulated polariton reflection curves. Attenuation of the lower branch of the anti-crossing is clearly observed. The inset in part (d) indicates the NNoPS of the same system without the J-aggregate. 
There is a qualitative agreement between the simulation results and the experimental results. Because of the discrepancies such as optical constants and grating profiles used in simulations and actual samples, there is a small difference in the dispersion curve of the coupled system. The upper band edge shifts from 583.4 to $584.8 \mathrm{~nm}$ and finally to $578.4 \mathrm{~nm}$ toward the blue as the groove depth increases from 5 to $10 \mathrm{~nm}$ and then to $15 \mathrm{~nm}$. The lower band edge shifts from 606.7 to $608.6 \mathrm{~nm}$ and then to $615.7 \mathrm{~nm}$ toward the red. In all cases, j-aggregate absorption line lies between the band edges. However, there is still a considerable amount of SPP modes inside the band gap [Fig. 2]; hence SPP-Exciton interaction is still possible for 5 and $10 \mathrm{~nm}$ cases. Shifting of upper and lower band edges increases the band gap energy from 81.6 to $83.3 \mathrm{meV}$ and finally to $129.9 \mathrm{meV}$. The increase of $E_{\mathrm{pg}}$ is confirmed by simulations. For the grating groove depth of $5 \mathrm{~nm}$, the value of the gap energy is $63.7 \mathrm{meV}$ and increases from 81.3 to $125.8 \mathrm{meV}$ when the groove depths are 10 and $15 \mathrm{~nm}$, respectively. Anti-crossing in 5 and $10 \mathrm{~nm}$ cases are 40 and $50 \mathrm{meV}$, respectively.

Attenuation of the lower branch of the anti-crossing is more clearly observed in the NNoPS data presented in Figs. 3(c) and 3(d). Figure 3(c) shows the experimentally obtained data and Fig. $3(\mathrm{~d})$ shows the simulation results with the inset showing the NNoPS data of the same system loaded with PVA minus J-aggregate. Anti-crossing is observed as two peaks on both sides of the J-aggregate resonance wavelength marked with a solid line at $590 \mathrm{~nm}$. A peak related to the lower branch of the anti-crossing is located at around $600 \mathrm{~nm}$. As the groove depth varies from 5 to $15 \mathrm{~nm}$, this peak attenuates since the number of the available plasmonic states in the band gap region decreases. The peak at around $580 \mathrm{~nm}$ belonging to the grating with the groove depth of $15 \mathrm{~nm}$ is actually the upper band edge and can be seen in the inset. On the other hand, the peaks at around $585 \mathrm{~nm}$ belonging to gratings with the groove depths of 5 and $10 \mathrm{~nm}$ are the upper branches of the anticrossing; they are not present in the case of the bare grating. Near the band edges, where the group velocity of the SPP modes are slowing down, NNoPS values rise; hence the effective amplitude of the electric field increases. Dye molecules have more probability to interact with the SPP modes [17]. Attenuation of the lower branch of the anti-crossing is a clear signal showing effectively suppression of the plasmon-exciton coupling inside the band gap region.

In conclusion, we have experimentally and theoretically demonstrated that plasmon-exciton coupling can be controlled by engineering the band gap of the plasmonic crystal. This leads to the observation that coupling is suppressed when the molecular resonance energy level is inside the band gap region along $\Gamma$ direction, and it is observed outside the band gap region. Thus a directional suppression of SPP-exciton interaction is achieved. Molecular resonance of the J-aggregate dye has been kept constant during all the experiments while the $E_{\mathrm{pg}}$ has been effectively controlled by the grating groove depth. This approach allows the study of plasmonexciton coupling at different regions of the band gap. The strength of the coupling inside and outside the band gap has been measured through the strength of Rabi splitting. The results indicate that the strength of the coupling between plasmons and excitons can be effectively controlled by plasmonic band gap engineering; therefore the coupled system can be used in plasmonic devices and in understanding light-matter interaction on plasmonic crystals.

This work has been supported by TUBITAK through grants 110T589 and 110T790.

\section{References}

1. Y. Kitamura and S. Murakami, Phys. Rev. B 88, 045406 (2013)

2. W. L. Barnes, T. W. Preist, S. C. Kitson, J. R. Sambles, N. P. K. Cotter, and D. J. Nash, Phys. Rev. B 51, 11164 (1995).

3. I. Avrutsky, Phys. Rev. B 70, 155416 (2004).

4. A. Kocabas, S. S. Senlik, and A. Aydinli, Phys. Rev. B 77, 195130 (2008).

5. A. Kocabas, S. S. Senlik, and A. Aydinli, Phys. Rev. Lett. 102, 063901 (2009).

6. W. L. Barnes, T. W. Preist, S. C. Kitson, and J. R. Sambles, Phys. Rev. B 54, 6227 (1996).

7. R. H. Ritchie, E. T. Arakawa, J. J. Cowan, and R. N. Hamm, Phys. Rev. Lett. 21, 1530 (1968).

8. D. L. Mills, Phys. Rev. B 15, 3097 (1977).

9. G. Granet and B. Guizal, J. Opt. Soc. Am. A 13, 1019 (1996).

10. J. Chandezon, M. T. Dupuis, G. Cornet, and D. Maystre, J. Opt. Soc. Am. 72, 839 (1982).

11. A. Kocabas, G. Ertas, S. S. Senlik, and A. Aydinli, Opt. Express 16, 12469 (2008).

12. T. Okamoto, F. H'Dhili, and S. Kawata, Appl. Phys. Lett. 85, 3968 (2004).

13. S. C. Kitson, W. L. Barnes, and J. R. Sambles, Opt. Commun. 122, 147 (1996).

14. N. T. Fofang, T.-H. Park, O. Neumann, N. A. Mirin, P. Nordlander, and N. J. Halas, Nano Lett. 8, 3481 (2008).

15. S. Balci, A. Kocabas, C. Kocabas, and A. Aydinli, Appl. Phys. Lett. 97, 131103 (2010).

16. J. R. Tischler, M. S. Bradley, Q. Zhang, T. Atay, A. Nurmikko, and V. Bulovic, Coupled States Excit. Photons Plasmons Org. Struct. 8, 94 (2007)

17. S. Balci, C. Kocabas, S. Ates, E. Karademir, O. Salihoglu, and A. Aydinli, Phys. Rev. B 86, 235402 (2012).

18. Y. B. Zheng, B. K. Juluri, L. L. Jensen, D. Ahmed, M. Lu, L. Jensen, and T. J. Huang, Adv. Mater. 22, 3603 (2010).

19. D. G. Lidzey, D. D. C. Bradley, A. Armitage, S. Walker, and M. S. Skolnick, Science 288, 1620 (2000).

20. D. G. Lidzey, D. D. C. Bradley, T. Virgili, A. Armitage, M. S. Skolnick, and S. Walker, Phys. Rev. Lett. 82, 3316 (1999).

21. J. Bellessa, C. Bonnand, J. C. Plenet, and J. Mugnier, Phys. Rev. Lett. 93, 036404 (2004).

22. C.Symonds, C. Bonnand,J. C.Plenet, A. Bréhier, R.Parashkov, J. S. Lauret, E. Deleporte, and J. Bellessa, New J. Phys. 10, 065017 (2008).

23. S. C. Kitson, W. L. Barnes, and J. R. Sambles, Phys. Rev. Lett. 77, 2670 (1996).

24. E. D. Palik, Handbook of Optical Constants of Solids (Academic, 1991) 